Accelerator Division

Alternating Gradient Synchrotron Department

BROOKHAVEN NATIONAL LABORATORY

Upton, New York 11973

Technical Note

AGS/AD/Tech. Note No. 402

\title{
UNIX UPGRADE FOR AGS CONTROLS WORKSTATIONS
}

J. T. Morris

October 11, 1994 


\section{Unix Upgrade for AGS Controls Workstations}

\section{Introduction}

The console computers now in use in the AGS Control System are all Hewlett-Packard/Apollo workstations which run the proprietary Domain/OS operating system. The Domain/OS computers, which are being discontinued by HP, have fallen behind other workstations in performance. In order to improve the performance of the console computers and provide a path for growth, plans are being made to move the Control System software to one of the more competitive Unix workstation platforms.

This document describes some of the reasons for moving away from Domain/OS. It briefly explores some of the major issues involved in the conversion of Control System software to run under a new operating system. It then presents plans for making a transition to new Unix workstation platforms.

\section{Problems With Apollo Domain/OS}

The Apollo Domain/OS was an innovative and technically very successful operating system. However, Apollo never became a big player in the workstation marketplace and in 1990, Apollo was bought out by Hewlett-Packard. HP made only modest developments in the Domain product line before announcing that the line would be discontinued. Domain/OS workstations and accessories will be available on the second hand market for a while, but the cost of maintaining the Control System on the Domain operating system will escalate with time. Expansion of the Control System will eventually become impossible.

A price is already being paid for the continued use of the Domain/OS operating system.

1) Domain workstations are expensive for the computing power that they provide. Domain 425 series workstations cost about the same as similarly equipped RISC workstations that have several times more processing power.

2) Commercially available software is often not supported on the Domain operating system. The same is true of public domain software. There is no incentive for software providers to port software to an operating system that is soon to be obsolete. LabViews and Mosaic are examples of two products that must be run on non-Domain platforms.

3) $\mathrm{X}$ performance on Domain/OS is weak. Domain was designed with its own proprietary methods for graphics and windowing. $X$ support was added later. $X$ puts great demands on the resources of any workstation, but there are some slowness problems that are peculiar to the Domain implementation. Since the Domain product line is being discontinued, HP is not aggressive in correcting Domain/OS problems. 
4) Problems have surfaced in the use of Domain's file transfer protocol on Ethernet and in Domain's implementation of the Address Resolution Protocol (arp). Both of these problems are acknowledged by HP but are not being fixed. In each case, the Controls Group has been forced to implement local system administration procedures to work around the weaknesses in the operating system.

\section{Software Conversion}

The demise of the Apollo Domain/OS operating system has been long anticipated by the Controls Group. The planning for a transition to a Unix platform began in 1991. The conversion task is not an easy one. There are more than 500000 lines of source code in the AGS Control System source code control system. There are approximately 45 active user applications, 13 server applications, and 18 miscellaneous support applications. Much of this code was originally written to use Apollo's proprietary calls for graphics, network communication, and general system services. The primary goal of the Control System conversion is to convert from these Apollo proprietary methods to standards that can be supported on a variety of platforms. For practical reasons, a single primary Control System platform and operating system will most likely be selected. However, the use of standards is the real key to the conversion effort. In fact, since most Unix standards are supported under Domain/OS, the Controls Group has been able to make substantial progress BEFORE selecting a target operating system.

The most visible part of the conversion effort has been the move from the Apollo Dialog user interface to the Motif and X Window standards. X and Motif are industry standards that are supported on almost all workstation platforms, including Domain. Roughly half of the Control System applications have been converted to the $\mathrm{X}$ user interface.

Network communication has been another major area of effort. The conversion from the Domain 'mailbox' communication protocol to the widely accepted standards of RPC and UDP is nearly completed. In the fall of 1992, a version of the Apollo station code was released which used the RPC/UDP protocol. Communication to older Relway stations was converted to RPC/UDP the following year. The final testing and release of RPC/UDP versions of all other Control System servers is now being planned.

In addition to the two areas mentioned above, Apollo proprietary system calls have been used in Control System software for a variety of services including file access, file protection, asynchronous event handling, time management, error handling, and multitasking. Work has begun on converting these calls to Unix standard equivalents, but the job is not yet done.

Complete software portability can only be demonstrated by compiling and running the software on multiple platforms. We are not yet able to run any Control System applications on a Unix workstation. However, the UI user interface library and qg graphics library have each been ported to a Sun platform. Work is now beginning on porting the Control System interface software to a Sun. Prototype applications are expected to be available on Unix platforms by early 1995. 


\section{Introduction of Unix Workstations}

The interdependence of hardware and software can make a smooth transition to a new operating system difficult. However, the client/server mechanism of the $\mathrm{X}$ window system will be used to ease the transition.

The $\mathrm{X}$ client/server model allows an application to run on one workstation with its display on another. New Unix console workstations can be introduced into the controls network prior to the complete conversion of applications. Applications can continue to run on Domain workstation platforms, while the user interface is displayed on the Unix workstation screen. Even in this configuration, the user sitting at the Unix workstation should experience some improvement in performance due to the improved $X$ server performance on the new machine. When an application is fully converted, it can run directly on the Unix workstation. This move can be made on an application-by-application basis.

The $\mathrm{X}$ client/server approach will be implemented in the main control room by introducing one Unix workstation as the "A node" at each console. One HP/Apollo 425t workstation will remain at each console. Initially, most applications will be running on the Apollo machine but displaying on the Unix workstation screen. The Apollo $425 \mathrm{t}$ will also be used to run any applications that have not yet been converted to an $\mathrm{X}$ interface.

Unix workstations will also be installed in control rooms in the field such as Linac, Tandem, and Booster and AGS Main Magnet and RF facilities. Users at these locations will run applications on designated Apollo server machines to display on their Unix workstations. Since these remote users will be working exclusively on a non-Domain Unix workstation, applications that are not converted to an $\mathrm{X}$ interface will not be available at these consoles. Therefore, all required applications must be converted to an $\mathrm{X}$ user interface before the Domain workstations at these locations can be replaced.

\section{Future Conversion Plans}

Once Unix workstations have been integrated into the AGS Control System, a second phase of Unix conversion work can begin. One goal for this second phase of conversion will be the replacement of the remaining HP/Apollo workstations at control room consoles. This change can be made when all controls applications have been converted to an $\mathrm{X}$ user interface. The future configuration of the control room consoles has yet to be determined but it may include $\mathrm{X}$ terminals and Unix workstations with multiple screens in addition to the traditional single screen workstation.

Another major conversion goal will be the replacement of HP/Apollo server workstations with Unix machines. Controls System servers provide a variety of services including database services, hardware interfaces, file access, and alarm system services. Some Control System servers provide a platform for running applications with displays on $\mathrm{X}$ terminals or other workstations. Each of these servers will have to be considered on an individual basis. 
The HP/Apollo machines that provide a platform for applications will be especially important during the transition period described in the preceding section. The demand for this service will decrease as applications are converted to run on Unix workstations. The HP/Apollo application servers will be retired. A demand for some number of Unix application servers is anticipated in the future to support users at $\mathrm{X}$ terminals.

File servers and alarm system servers should be relatively easy to move to Unix platforms. These do not depend on any resources that are particular to HP/Apollo machines. Database servers, however, depend on a licensed database management system. The database management system currently being used on HP/Apollo machines may be replaced by another database product on Unix platforms. Serious planning will be required before changes are made in the area of database management.

Control System servers that provide hardware interfaces will most likely not be directly converted to run on Unix workstations. Some of this functionality will be taken off workstations and put onto real time systems in the future. Other services, such as the interface to the Relway network, will be supported on Apollo workstations until the service is no longer needed. 